\title{
Decentralization of Services Through Three Tiers in Wireless Body Area Networks
}

\author{
Said Lakhal, and Zouhair Guennoun IEEE, Senior member
}

\begin{abstract}
The Wireless Body Area Network (WBAN) contains a set of sensors, placed in the patient's environment, to detect the vital signs and transmit the results towards the relevant services that interact in urgent cases. The present work exposes a functional WBAN architecture formed by three layers: closest, intermediate and farthest. The closest layer senses the chemical and biological signs. If the case is normal, or it can be locally regulated, the service stops there; else the intermediate service, namely the local hospital, is consulted to make treatments. Therefore, the patient will be transported into the hospital for examinations. If this condition is established the processus stopped there, otherwise, the request of help from an outside hospital becomes necessary. This request passes through a manager that supervises a network of hospitals and looks for a free place to welcome the patient. After the localization of a hospital, its coordinates will be forwarded into the customer hospital, for transporting the patient. The simulation results show that this design increases the patient's probability of healing and maximizes the use of the available resources, relative to the centralization of services at a single layer or at two layers.
\end{abstract}

Index Terms-WBAN, decentralization of services, centralization of services, probability of healing, resource optimization.

\section{INTRODUCTION}

T he WBAN is defined by the IEEE 802.15.6 and the IEEE 802.15.4j [1] as a collection of distributed sensors: around, on or in the human body to collect data. The sensed information is converted, stored, processed and transmitted towards the health service, to inform or request the intervention. This technology with a remote and automatic service, clean and easy, has revolutionized the services and infrastructure of hospitals, ambulances and homes.

The increased traffic via this network, prolongs the time for transferring data, to the concerned services. First aid may therefore arrive late; sometimes after the deterioration of the patient's condition and the desperation to heal. Consequently, wait time becomes an essential parameter to indicate the performance of WBAN. Occasionally, the consulted hospital can be saturated, so no intervention can be performed; while other hospitals offering the same services are free. This situation shows both: the limitation of the policy of centralizing services in a single resource, and the importance of optimizing the resources available in a network. Highlighting, the IEEE

Manuscript received November 25, 2019; revised April 9, 2020. Date of publication June 3, 2020. Date of current version June 3, 2020.

Authors are with the Research Team in Smart Communications-ERSC, E3S Research Center, EMI, Mohammed V University in Rabat, Morocco (e-mails: said.lakhal.rech@gmail.com,zouhair@emi.ac.ma).

Digital Object Identifier (DOI): 10.24138/jcomss.v16i2.955
802.15.6 standardization emphasized the importance of realtime communication and resource optimization, to save the patient health [1]. For instance, an emergency packet is sent to the health services to indicate the patient's critical condition, in order to intervene [2]. D.Ousmane et al. [3] studied the minimization of the communication delay, by proposing both: an architecture and a query processing algorithm that are combined with cloud services and statistical modeling techniques. As well, the problem of resource optimization had found its place in the literature [1], [4]. The contribution of [4] was based on that of [1], after developing a prioritized resource allocation algorithm, to share the limited communication channel between multiple WBANs.

In summary, minimizing the communication delay [1], [3], prioritizing the emergency package [2] or optimizing the communication resource [3] are important for serving the patient's life. For our part, we take advantage of the WBAN architecture to increase the patient's probability of recovery. For this goal, we design a functional WBAN architecture, formed by three layers: closest, intermediate and farthest. Each one performs its tasks to enhance the patient's condition; in the case of the inability, it requests the help from the upper adjacent layer, and so, until the good health or the incapacity of the global service. The tasks of these layers are distributed as follows: the closest layer is occupied to collect the vital signs using sensors, to analyze the results by smart devices, and to regularize the local cases using actuators. When this layer fails to solve the problem, the middle layer takes care of the patient. First, an equipped ambulance, accompanied by a health team, makes a site visit of the patient. After the preliminary treatments, if the patient's condition is regulated, the service stops there; otherwise, the patient will be transported to the local hospital; in order to make exhaustive diagnoses and cares. Despite its efforts, sometimes, this hospital remains helpless against some situations. Therefore, it will be obligated to ask for an outside help. The request for help is intercepted by the hospital network manager, which is looking for a free place in the network. Once this place has been found, the coordinates of the hospital concerned will be transmitted to the intermediate layer, to transport the patient to the said hospital. Via this architecture, we decentralize services through three layers. This is why, we are improving the patient's probability of recovery rather than centralizing services at one or at two layers.

The rest of this work is organized as follows: in section II, we present some related works based on the resource optimization and delay minimization. We process by levels 
in section III, to enhance the probability of healing and optimize the hospital network. In section IV, we model the probability of healing, and we analytically evaluate our model relative to centralization of services at one or at two levels. The simulation results are exposed in section V. Finally, we conclude in section VI.

\section{RELATED WORKS}

The optimization of energy, time and communication infrastructure had attracted enormous works. A hybrid autonomic resource provisioning framework is proposed in [5]. This approach combined between autonomic computing and queuing model. The simulation results showed an amelioration in terms of resource utilization and response time, by at least $26 \%$ and $49 \%$, respectively, compared with other methods. To study the effect of sensor communication on the energy consumption and throughput, the authors of [6] proposed two protocols: Optimized Cost Effective and Energy Efficient Routing protocol (OCER), as well as an extended-OCER. In another study [7], a scheme leverages Lagrangian duality theory is designed, to find efficient trade-off among energy consumption, network cost, and vital signs distortion, for delay sensitive transmission of medical data. The optimal trade-off between energy efficiency and QoS requirements, while providing 15\% savings the energy-cost-distortion utility function, compared to solutions based on equal bandwidth allocation. In order to maintain Mobile Data Collectors (MDC)-relay networks, the paper [8] proposed a self-organizing and an adaptive Dynamic Clustering Multiple MDC. This solution is based on dividing the network into well-delimited clusters, called Service Zones. The experimental performances improved the packet delivery ratio and end-to-end delay by up to $10 \%$. The energy consumption, energy balancing and network lifetime, each one by up to $51 \%$. A Superframe Overlapping Scheduling (SOS) based on beacon shifting and active superframe interleaving techniques used the IEEE 802.15.6 standard, was developed by [9]. The authors parameterized the required service rate and maximum packet inter-arrival time for each WBAN. The SOS conception impoved bandwidth utilization and guaranteed QoS, according to individual WBAN task deadlines. With the aim to study the relation between the data packet transmission delay and the system dynamics, as well as, the impact of the stability regions and bifurcation scenario, the work developed in [10], proposed a delayed e-epidemic model and explored the dynamic systems. The paper [11] summarized characteristics of Intermittently Connected Delay-Tolerant Wireless Sensor Networks (ICDT-WSNs) and their communication protocol requirements, also examined the communication protocols designed for WSNs in the recent years from the perspective of ICDT-WSNs. To solve the issues in reconstruction computation complexity of WBAN, the work of [12] developed compressed sensing for compressing and collecting signals in realtime. The evaluation of results showed that the improvement of real-time performance is contributed by the application of binary permutation block diagonal matrix. The security issues are treated in [13] to ensure the sensor data acquisition and transmission protocols. The main security features are described and compared in the context of a secure eHealthcare system. In the other side, the work developed in [14] proposed an energy efficient wireless telemonitoring scenario of heart patients through ZigBee, under variable load conditions. This method is compared with different percentage of duty cycle on the basis of energy consumption parameter.

The WBAN architecture has attracted the attention of several researchers [15], [16], [17]. L.Ahmed et al. [15] proposed an architecture for collecting and accessing large amount of data that is generated by medical sensor networks. This architecture overcame enormous challenges, and made easy information sharing, between healthcare professionals in normal and emergency situations. On the other side, A.Bandar and F.Hacene [16] detailed the features hardware and software for mobile health-monitoring platform.

These related works were aimed at minimizing the transmission delay and maximizing the resource use. In our approach, we emphasize the importance of the adopted architecture to increase the probability of healing and optimize the available resources of a hospital network.

\section{Our Approach And Functional Architecture}

Regarding their location to the patient, the overall service is made up of three layers: closest, intermediate and farthest. When a lower service fails to establish the patient's condition, it will ask for help from the upper adjacent service; until the regulation of the anomaly, or the inability of the whole service. In the next, the function of each level will be detailed.

\section{A. Closest Service}

1) Components: This service is the closest to the patient. Its principal components are: sensors, hub, smart device and regulators (Fig.1). The sensors detect vital signs, convert them

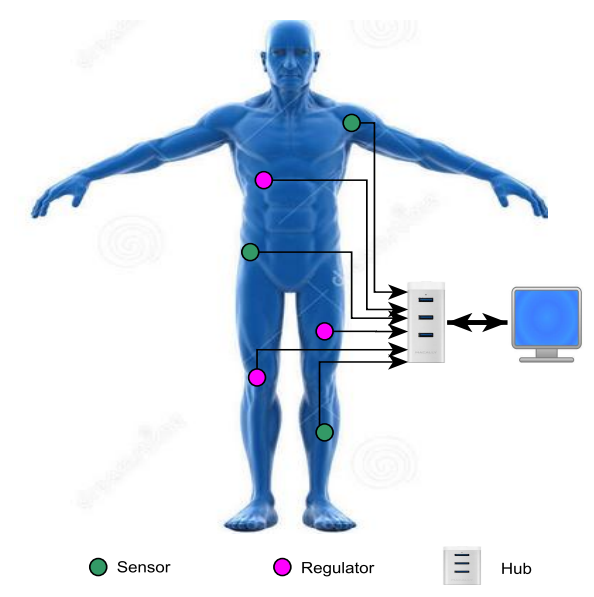

Fig. 1. BAN

into physical signs and finally transmit the signals obtained to the hub. This latter, collects all signals and transmits the results as a vector to the smart device, to make treatments, based on the following model: 
2) Operation of the Smart Device: Let $W$ be the received vector, such as:

$$
W=\left(w_{1}, ., w_{j}, ., w_{n}\right)
$$

Each component $w_{j}$ has a measurement unit $u_{j}$. Thus, we put:

$$
w_{j}=v_{j} \times u_{j}
$$

Where $v_{j}$ is a numerical value. The normal state relating to a vital sign $j$, is indicated by the belonging of $w_{j}$ to a confidence interval:

$$
C I_{j}=\left[\min _{j} \times u_{j}, \max _{j} \times u_{j}\right]
$$

The overall normal state is ensured by the belonging of each $w_{j}$ to its confidence interval. To specify this membership, we calculate a scalar $S_{j}$ which is unitless:

$$
\begin{aligned}
S_{j} & =\frac{\left(\max _{j} \times u_{j}-v_{j} \times u_{j}\right)\left(v_{j} \times u_{j}-\min _{j} \times u_{j}\right)}{\left(\max _{j} \times u_{j}-\min _{j} \times u_{j}\right)^{2}} \\
& =\frac{\left(\max _{j}-v_{j}\right)\left(v_{j}-\min _{j}\right)}{\left(\max _{j}-\min _{j}\right)^{2}}
\end{aligned}
$$

As a result, we have the following equivalences:

$$
w_{j} \in C I_{j} \Leftrightarrow S_{j} \geq 0 \Leftrightarrow\left|S_{j}\right|=S_{j} \Leftrightarrow\left|S_{j}\right|-S_{j}=0
$$

Then, we can define an elementary indicator $e_{j}$ based on $S_{j}$ :

$$
e_{j}=\left|S_{j}\right|-S_{j}
$$

Finally, we establish a global indicator:

$$
g=\sum_{j=1}^{n} e_{j}
$$

While each $e_{j}$ is unitless, $g$ is well defined. Then, we have:

Corollary 1: The patient is in good overall condition if and only if $g=0$.

If $\mathbf{g}$ is not zero, an abnormal state requires an intervention. To specify the service involved, the smart device uses the vector $\mathrm{W}$, whose components will be classified into two families: L and I, for locally adjustable vital signs and others intermediately, respectively. As a result, the vector $W$ becomes:

$$
W=(L, I), \quad L=\left(w_{1}, \ldots, w_{i}\right), \quad I=\left(w_{i+1}, \ldots, w_{n}\right) .
$$

Accordingly, the smart device processes as follows:

1) Calculate the value of $g$ : if $g=0$, there is no problem, else;

2) Browse the components of $L$ : if there is a non-zero component, the adjusted value and the concerned regulator must be specified, else;

3) Ask for help from the intermediate service (Fig.3).

\section{B. Intermediate Service}

The intermediate service is the local hospital that monitors a group of patients around its territory. At this level, a service categorization is performed. If the state can be intermediately treated and the hospital is not yet saturated, a visit of the patient will be carried out. After that, if the good state is established, the service is completed here; if not, the move of the patient into the hospital becomes necessary, to perform exams and treatments. Following these operations, in the case of regulation of the state of health, the task is accomplished here. Otherwise, a rescue message is forwarded to the hospital network manager. (Fig. 3).

\section{Farthest Service}

The hospital network is administered by a manager that communicates with all hospitals, in order to provide service to the patient (Fig.2)

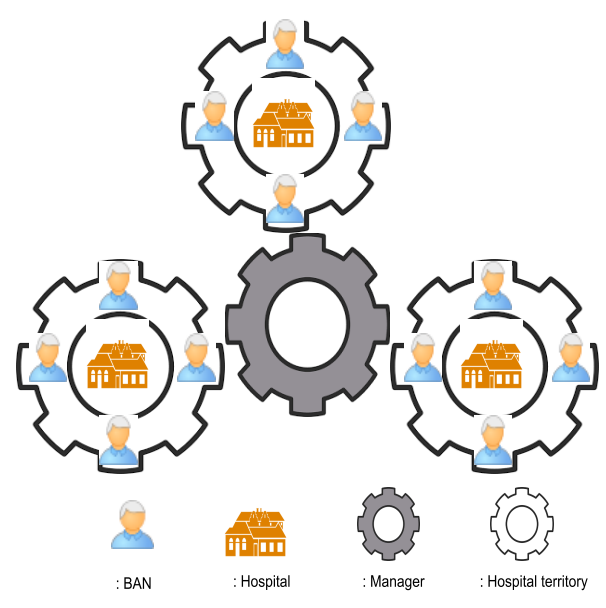

Fig. 2. Operation of the hospital network manager

After receiving a help request from the intermediate service, the network manager looks for a free place in a set of hospitals, to welcome the patient. If such place is found, a message indicating the receptive hospital coordinates will be sent to the intermediate service, to move the patient. Otherwise, a message mentioning the absence of service is transmitted, and the service is stopped there (Fig.3).

\section{PERFormanCES}

\section{A. Probabilities of Healing}

Simply, our model proceeds as follows:

1) If the actuators adjust the patient's condition, that is fine; otherwise,

2) the patient will be treated by local hospital. If the latter gives a good result, the service stops there; else,

3) the patient will be greeted by the hospital network manager which selects an available hospital, to treat the patient's anomaly. 
To identify the layer that brings good health to the patient, we define three random variables: $X_{1}, X_{2}$ and $X_{3}$ that correspond to regulator, local hospital and hospital network, respectively. Each of them follows a Bernoulli law, thus we have equation (9):

$$
X_{i}= \begin{cases}1 & \text { if the patient cures at level i, } \\ 0 & \text { if not, } \quad \forall i \in\{1,2,3\}\end{cases}
$$

Therefore, we can write:

$p\left(X_{i}=1\right)=p_{i}, p\left(X_{i}=0\right)=1-p_{i}$, with $p_{i} \in[0,1]$.

The probability of healing by our model or decentralization of services, can be defined as follows:

$$
\begin{aligned}
p_{\text {ho }}= & p\left(X_{1}=1\right)+p\left(X_{1}=0, X_{2}=1\right) \\
& +p\left(X_{1}=0, X_{2}=0, X_{3}=1\right) \\
= & p_{1}+\left(1-p_{1}\right) p_{2}+\left(1-p_{1}\right)\left(1-p_{2}\right) p_{3}
\end{aligned}
$$

\section{B. Comparison between Centralization and Decentralization of Services}

1) Centralization at one level:

- At the $1^{\text {st }}$ level:

The probability of healing at this level is $p_{1}$.

$$
\begin{aligned}
p_{\text {ho }}-p_{1} & =\left(1-p_{1}\right) p_{2}+\left(1-p_{1}\right)\left(1-p_{2}\right) p_{3} \\
& =\left(1-p_{1}\right)\left(p_{2}+\left(1-p_{2}\right) p_{3}\right) \\
& =\left(1-p_{1}\right)\left(p_{3}+\left(1-p_{3}\right) p_{2}\right) \geq 0
\end{aligned}
$$

- At the $2^{\text {nd }}$ level:

The probability of healing at this level is $p_{2}$.

$$
\begin{aligned}
p_{h o}-p_{2} & =p_{1}+\left(1-p_{1}\right) p_{2}-p_{2}+\left(1-p_{1}\right)\left(1-p_{2}\right) p_{3} \\
& =p_{1}+\left(1-p_{1}-1\right) p_{2}+\left(1-p_{1}\right)\left(1-p_{2}\right) p_{3} \\
& =p_{1}-p_{1} p_{2}+\left(1-p_{1}\right)\left(1-p_{2}\right) p_{3} \\
& =\left(1-p_{2}\right)\left(p_{1}+\left(1-p_{1}\right) p_{3}\right) \\
& =\left(1-p_{2}\right)\left(p_{3}+\left(1-p_{3}\right) p_{1}\right) \geq 0
\end{aligned}
$$

- At the $3^{\text {rd }}$ level:

The probability of healing at this level is $p_{3}$.

$$
\begin{aligned}
p_{h o}-p_{3} & =p_{1}+\left(1-p_{1}\right) p_{2}+\left(1-p_{1}\right)\left(1-p_{2}\right) p_{3}-p_{3} \\
& =p_{1}+\left(1-p_{1}\right) p_{2}+\left[\left(1-p_{1}\right)\left(1-p_{2}\right)-1\right] p_{3} \\
& =p_{1}+\left(1-p_{1}\right) p_{2}+\left[\left(1-p_{1}\right)\left(1-p_{2}\right)-1\right] p_{3} \\
& =p_{1}+\left(1-p_{1}\right) p_{2}+\left[-p_{1}-p_{2}+p_{1} p_{2}\right] p_{3} \\
& =p_{1}+p_{2}-p_{1} p_{2}-p_{1} p_{3}-p_{2} p_{3}+p_{1} p_{2} p_{3} \\
& =p_{1}-p_{1} p_{3}+p_{2}-p_{2} p_{3}-p_{1} p_{2}+p_{1} p_{2} p_{3} \\
& =p_{1}\left(1-p_{3}\right)+p_{2}\left(1-p_{3}\right)+p_{1} p_{2}\left(-1+p_{3}\right) \\
& =\left(1-p_{3}\right)\left(p_{1}+p_{2}-p_{1} p_{2}\right) \\
& =\left(1-p_{3}\right)\left(p_{1}+\left(1-p_{1}\right) p_{2}\right) \\
& =\left(1-p_{3}\right)\left(p_{2}+\left(1-p_{2}\right) p_{1}\right) \geq 0
\end{aligned}
$$

From equations : (11), (12) and (13), we deduce that:

$$
\begin{aligned}
& p_{h o}-p_{i}=\left(1-p_{i}\right)\left(p_{j}+\left(1-p_{j}\right) p_{k}\right) \geq 0, \\
& \forall i \neq j \neq k, \quad \text { and } \quad(i, j, k) \in\{1,2,3\}^{3}
\end{aligned}
$$

- Expression of $p_{h o}$ as a function of $p_{i}, \forall i \in\{1,2,3\}$ : Form equation (14), and after simplification, we have:

$$
\begin{aligned}
& p_{h o}=\left[p_{j}+\left(1-p_{j}\right) p_{k}\right]+\left[\left(1-p_{j}\right)\left(1-p_{k}\right)\right] \mathbf{p}_{\mathbf{i}} \\
& \forall i \neq j \neq k, \quad \text { and } \quad(i, j, k) \in\{1,2,3\}^{3}
\end{aligned}
$$

2) Centralization at Two Levels:

- At the $1^{\text {st }}$ and $2^{\text {nd }}$ level:

The probability of healing at these levels is: $p_{12}=p_{1}+\left(1-p_{1}\right) p_{2}$.

$$
\begin{aligned}
p_{\text {ho }}-p_{12} & =p_{1}+\left(1-p_{1}\right) p_{2}+\left(1-p_{1}\right)\left(1-p_{2}\right) p_{3} \\
& -p_{1}-\left(1-p_{1}\right) p_{2} \\
& =p_{3}\left(1-p_{1}\right)\left(1-p_{2}\right) \geq 0 .
\end{aligned}
$$

- At the $1^{\text {st }}$ and $3^{\text {rd }}$ level:

The probability of healing at these levels is: $p_{13}=p_{1}+\left(1-p_{1}\right) p_{3}$.

$$
\begin{aligned}
p_{h o}-p_{13}= & p_{1}+\left(1-p_{1}\right) p_{2}+\left(1-p_{1}\right)\left(1-p_{2}\right) p_{3} \\
& -p_{1}-\left(1-p_{1}\right) p_{3} \\
= & \left(1-p_{1}\right) p_{2}+\left(1-p_{1}\right)\left(1-p_{2}\right) p_{3} \\
& -\left(1-p_{1}\right) p_{3} \\
= & \left(1-p_{1}\right) p_{2}+\left(1-p_{2}-1\right)\left(1-p_{1}\right) p_{3} \\
= & \left(1-p_{1}\right) p_{2}-p_{2}\left(1-p_{1}\right) p_{3} \\
= & p_{2}\left(1-p_{1}\right)\left(1-p_{3}\right) \geq 0 .
\end{aligned}
$$

- At the $2^{\text {nd }}$ and $3^{\text {rd }}$ level:

The probability of healing at these levels is:

$p_{23}=p_{2}+\left(1-p_{2}\right) p_{3}$.

$$
\begin{aligned}
p_{\text {ho }}-p_{23}= & p_{1}+\left(1-p_{1}\right) p_{2}+\left(1-p_{1}\right)\left(1-p_{2}\right) p_{3} \\
& -p_{2}-\left(1-p_{2}\right) p_{3} \\
= & p_{1}+\left(1-p_{1}\right) p_{2}-p_{2} \\
& +\left(1-p_{1}-1\right)\left(1-p_{2}\right) p_{3} \\
= & p_{1}-p_{1} p_{2}-p_{1}\left(1-p_{2}\right) p_{3} \\
= & p_{1}\left(1-p_{2}\right)-p_{1}\left(1-p_{2}\right) p_{3} \\
= & p_{1}\left(1-p_{2}\right)\left(1-p_{3}\right) \geq 0 .
\end{aligned}
$$

From equations: (16), (17) and (18), we deduce that:

$$
\begin{aligned}
& p_{i j}=p_{i}+\left(1-p_{i}\right) p_{j}, \\
& p_{h o}-p_{i j}=p_{k}\left(1-p_{i}\right)\left(1-p_{j}\right) \geq 0, \\
& \forall i \neq j \neq k, \quad \text { and } \quad(i, j, k) \in\{1,2,3\}^{3}
\end{aligned}
$$

- Expression of $p_{h o}$ as a function of $p_{i j}, \forall i \neq j$ and $(i, j) \in$ $\{1,2,3\}^{2}$.

From equation (19) and after simplification, we can write:

$$
\begin{aligned}
& p_{h o}=p_{k}+\left(1-p_{k}\right) \mathbf{p}_{\mathbf{i j}} \\
& \forall i \neq j \neq k, \quad \text { and } \quad(i, j, k) \in\{1,2,3\}^{3}
\end{aligned}
$$

Analytically, we have established that the decentralization of services is better than its centralization at one or two levels. 


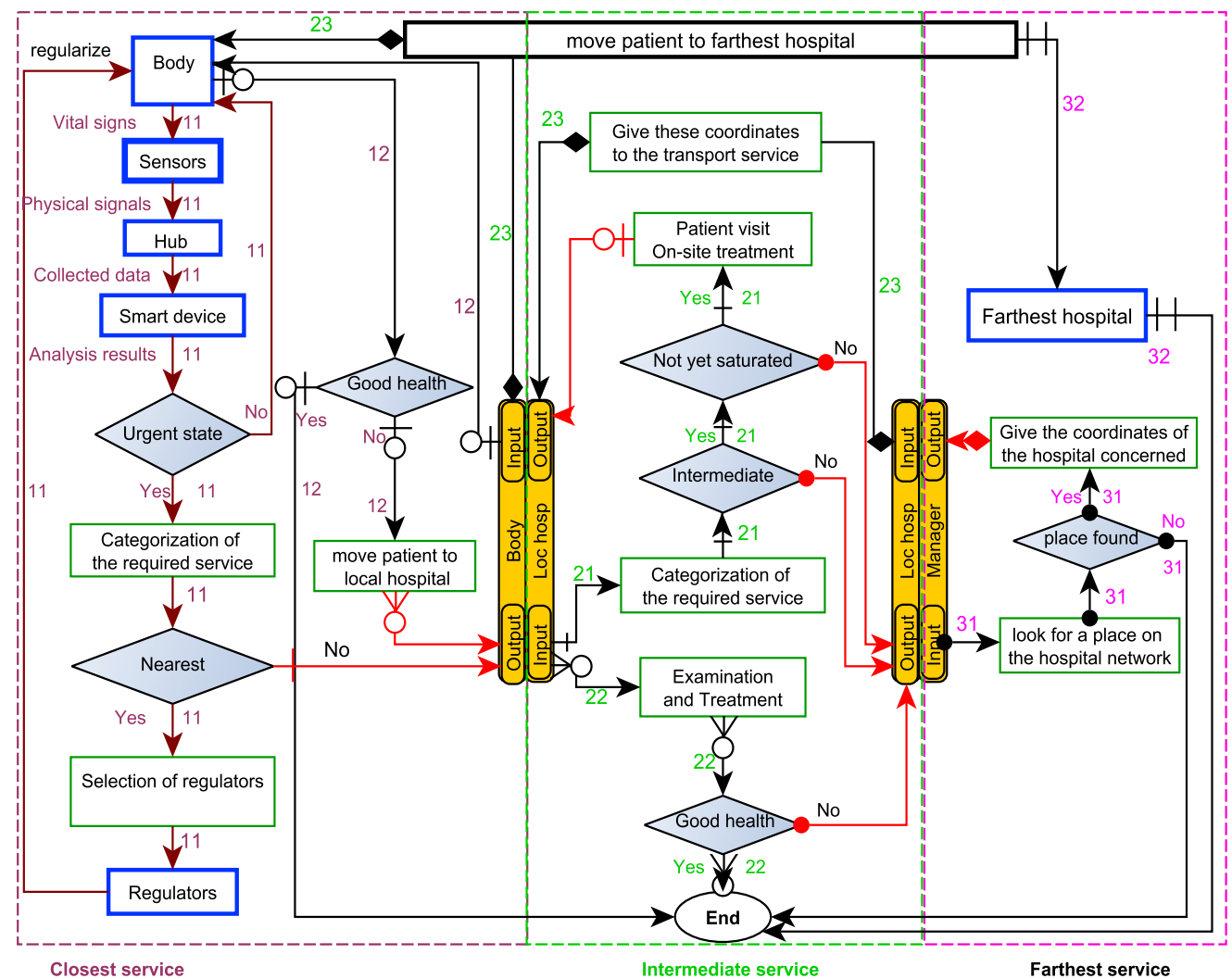

Fig. 3. Functional architecture: closest layer identified by number $1\left(11: 1^{\text {st }}\right.$ time, $12: 2^{\text {nd }}$ time $)$, intermidiate layer indicated by number $2\left(21: 1^{\text {st }}\right.$ time, 22 : $2^{\text {nd }}$ time, $23: 3^{\text {rd }}$ time $)$ and farthest layer represented by number $3\left(31: 1^{\text {st }}\right.$ time, $32: 2^{\text {nd }}$ time $)$.

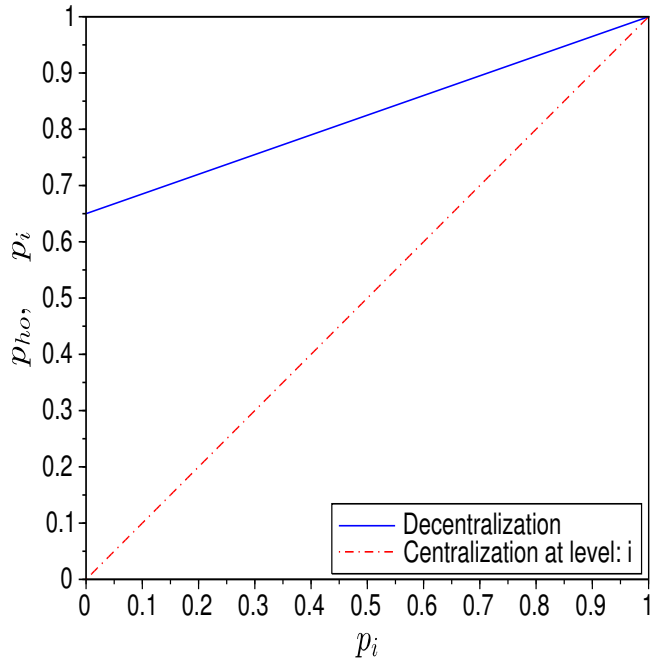

(a) Centralization of services at one level

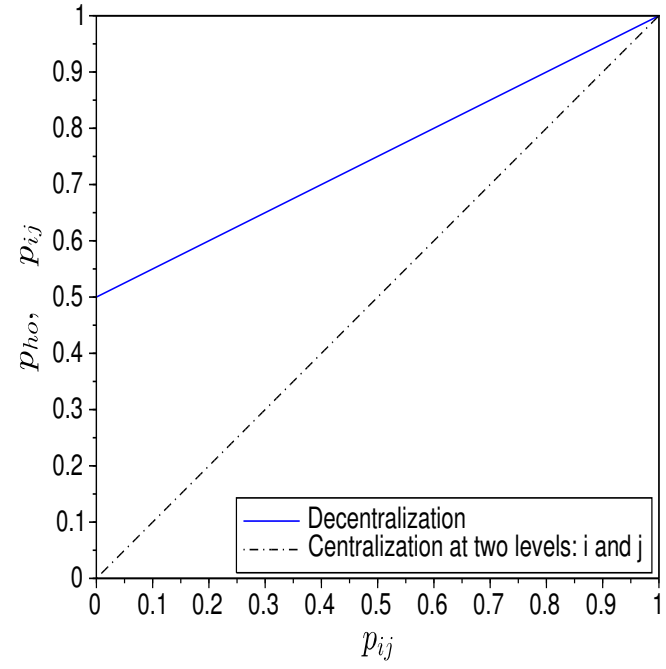

(b) Centralization of services at two levels

Fig. 4. Comparison between decentralization and centralization of services

\section{Simulations}

Currently, we graph the results, to visually show the performance of our model. This graphical representation is based on equation (15) and (20), to make the comparison with the decentralization of services and their centralization at one level or at two levels, respectively.

The comparison between the decentralization of services 
and their concentration in a single level is illustrated in sub figure 4.(a). To do this, we choose numerical values within the interval $[0,1]$, for the probability of healing $p_{i}$ at the level $\mathrm{i}$, and we represent them on the $\mathrm{x}$-axis. The $\mathrm{y}$-axis is devoted to the enumeration of the values of $p_{i}$ and the probability of healing by our model $p_{h o}$. In addition, to show the difference between decentralization and centralization at two levels, we draw the curves of sub figure 4.(b): on the $\mathrm{x}$-axis are placed the values of the probability of healing $p_{i j}$ for level $\mathrm{i}$ and $\mathrm{j}$ together. On the y-axis, we distribute the values of $p_{h o}$ and $p_{i j}$. We can easily notice that the distribution of services through three levels is always better than their centralization at a single level or at two levels. This result is interpreted by the collaboration of three services to meet the needs of a patient, more efficiently than a single level or even two.

\section{CONCLUSION}

In this work, we have designed a functional WBAN architecture that is formed by three layers named based on their locations in relation to the patient: closest, intermediate and farthest. The closest detects the vital signs through the sensors that are placed on the body. These signs are converted into physical signals, in order to transmit them to an intelligent device, which performs treatments, to categorize the state of health. In the event of a locally treatable anomaly, the regulators concerned and the adjusted values are specified. Otherwise, a rescue message will be sent to the intermediate layer, namely the local hospital, which will consult the patient on site, by using a mobile service, to carry out preliminary treatments. In case of regulation, the service stops there; in the other case, the patient will be transported to the hospital, to make the possible diagnoses and treatments. After these efforts, the patient's condition can be adjusted, as it cannot be: in the unpleasant case, the intermediate service will ask for the help from the farthest layer, namely the hospital network manager, to find an available hospital to receive the patient. Once such a hospital is found, its coordinates will be sent to the intermediate service that will take care of the transportation of the patient to the said hospital. Such an approach makes it possible to strengthen the patient's chances of recovery and also to maximize the exploitation of the hospital resources available on a network of hospitals.

\section{REFERENCES}

[1] M.Samaneh, A.Mehran, L.Justin, and J.Abbas, "Wireless Body Area Networks: A Survey", IEEE Communications Surveys and Tutorials, 16, 1658-1686 (2014). doi: 10.1109/SURV.2013.121313.00064

[2] B.Nourchene, C.F.Lamia and K.Lotfi, "WBAN data scheduling and aggregation under WBAN/WLAN healthcare network", Ad Hoc Networks, 25,251-262 (2015). doi: 10.1016/j.adhoc.2014.10.017

[3] D.Ousmane, J.P.C.R.Joel , S.Mbaye and N.Jianwei, "Real-time query processing optimization for cloud-based wireless body area networks", Information Sciences, 284, 84-94(2014). doi: 10.1016/j.ins.2014.03.081

[4] K.Seungku and K.S.Byung, "A prioritized resource allocation algorithm for multiple wireless body area networks", Wireless Networks, 23,727735 (2017). doi: 10.1007/s11276-015-1163-1

[5] B.Tushar and C.S.Subhash, "Cloud-WBAN: An experimental framework for Cloud-enabledWireless Body Area Network with efficient virtua resource utilization, Sustainable Computing", Informatics and Systems, 20, 14-33 ( 2018). doi: 10.1016/j.suscom.2018.08.008
[6] K.Navneet and S.Sukhwinder, "Optimized Cost Effective and Energy Efficient Routing Protocol for Wireless Body Area Networks", Ad Hoc Networks, 61, 65-84 (2017). doi: 10.1016/j.adhoc.2017.03.008

[7] A.Alaa , M.Amr, C.F.Carla and E.Tarek, "Distributed in-network processing and resource optimization over mobilehealth systems", Journal of Network and Computer Applications, 82, 65-76 (2017). doi: 10.1016/j.jnca.2017.01.014

[8] A.Abdelrahman, H.Mohammad, A.Bamidele, J.Sohail, B.Ahcene and A.B.Hashem, "Dynamic clustering and management of mobile wireless sensor networks", Computer Networks, 117, 62-75 (2017). doi: 10.1016/j.comnet.2017.02.001

[9] R.C.Da, "A Real-time Streaming Control for Quality-of-Service Coexisting Wireless Body Area Networks", Applied Soft Computing, 68, 719-732 (2017). doi: 10.1016/j.asoc.2017.08.035

[10] K.U.Ranjit and K.Sangeeta, "Discrete and data packet delays as determinants of switching stability in wireless sensor networks", Applied Mathematical Modelling, 72, 513-536(2019). doi: 10.1016/j.apm.2019.03.028

[11] Ying and B.Radim, "A survey of protocols for Intermittently Connected Delay-Tolerant Wireless Sensor Networks", Journal of Network and Computer Applications, 41, 411-423(2014). doi: 10.1016/j.jnca.2013.10.003

[12] L.Dan, W.Qisong, Z.Yan, L.Xin, L.Jingyang and S.Jinwei, "FPGA-based real-time compressed sensing of multichannel EEGsignals for wireless body area networks", Biomedical Signal Processing and Control, 49, 221230(2019). doi: 10.1016/j.bspc.2018.12.019

[13] Y.Muhammad, S.Kashif, A.O.Mehmet, A.Haider, A.M.Jalal, I.Waseem and R.Imran, "Secure Sensors Data Acquisition and Communication Protection in eHealthcare: Review on State of the Art", Telematics and Informatics, 4,702-726(2018). doi: 10.1016/j.tele.2017.08.005

[14] P.Shashwat, K.Mayur, M.Amrita and K.Basant, "Energy Optimization of ZigBee based WBAN for Patient Monitoring", In Procedia Computer Science, 70, 414-420 (2015). doi: 10.1016/j.procs.2015.10.055

[15] L.Ahmed, H.Abdelkrim, B.Abdelmadjid, C.Yacine, "Healing on the cloud: Secure cloud architecture for medical wireless sensor networks", Future Generation Computer Systems, 55, 266-277(2016). doi: 10.1016/j.future.2015.01.009

[16] A.Bandar and F.Hacene, "A Mobile Wireless Body Area Network Platform", Journal of Computational Science, 5, 664-674 (2014). doi: 10.1016/j.jocs.2014.02.008

[17] S.Lakhal and Z.Guennoun, "The impacts of dynamic and static resource allocation in a wireless body area network", International Conference on Advanced Communication Technologies and Networking (CommNet18), doi: 0.1109/COMMNET.2018.8360257

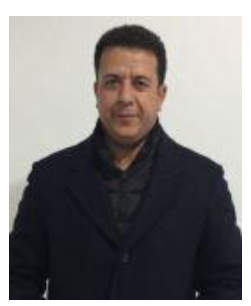

Said Lakhal obtained the diploma of application engineer in computer sciences in 1998, from the University Sidi Mohamed Ben Abdelah, Fes, Morocco. M.Sc. degree in modelization in 2006, from Mohammadia School of Engineering. He is currently a member of Team in Smart CommunicationsERSC, E3S Research Center, EMI, Mohammed V University in Rabat, Morocco. His current research interests are Computing, Cognitive Radio, WBAN, IoT, Algorithmic and Complexity.

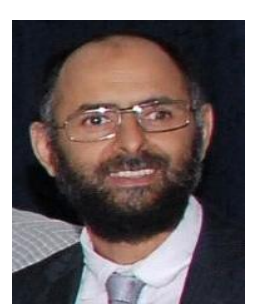

Zouhair Guennoun was born in Fès, Morocco in 1964. He received his engineering degree in Electronics and Telecommunications from the Electronics and Electrical Montefiore Institute, ULG Liege, Belgium in 1987; his M.Sc. degree in Communication Systems from the EMI School of Engineering, Rabat, Morocco in 1993; and his PhD degree from the same school in 1996. He visited the Centre for Communication Research (CCR) in Bristol University, UK, during the period of 1990-1994 to prepare a split $\mathrm{PhD}$. His fields of interest are digital signal processing, error control coding, speech and image processing, telecommunication systems, networks architecture and networks security. Prof. Guennoun is an IEEE senior member (member since 1990); and an ex-member of the Moroccan IEEE section executive committee. During 1988-1996 he worked as an Assistant Lecturer in the EMI School of engineering, and from 1996 he is working in the same school as a Professor Lecturer. Currently, he is in charge of the research team of Smart Communications-ERSC (formerly known as LEC) as part of the research center in engineering sustainable and smart systems at EMI, University Mohammed V in Rabat. 